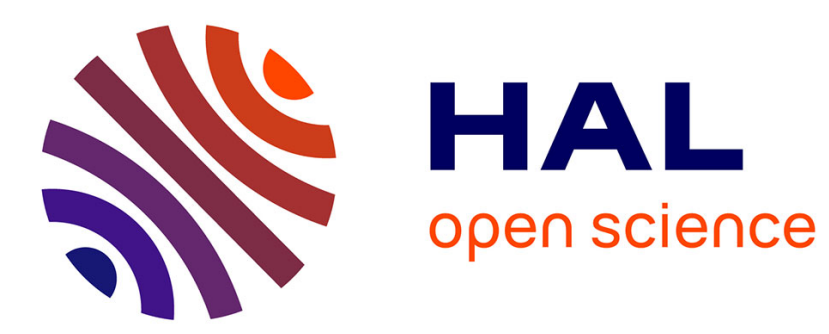

\title{
Effect of the matrix behavior on the damage of ethylene-propylene glass fiber reinforced composite subjected to high strain rate tension
}

Joseph Fitoussi, Michel Bocquet, Fodil Meraghni

\section{To cite this version:}

Joseph Fitoussi, Michel Bocquet, Fodil Meraghni. Effect of the matrix behavior on the damage of ethylene-propylene glass fiber reinforced composite subjected to high strain rate tension. Composites Part B: Engineering, 2012, 45, pp.1181-1191. 10.1016/j.compositesb.2012.06.011 . hal-00984755

\section{HAL Id: hal-00984755 \\ https://hal.science/hal-00984755}

Submitted on 30 Apr 2014

HAL is a multi-disciplinary open access archive for the deposit and dissemination of scientific research documents, whether they are published or not. The documents may come from teaching and research institutions in France or abroad, or from public or private research centers.
L'archive ouverte pluridisciplinaire HAL, est destinée au dépôt et à la diffusion de documents scientifiques de niveau recherche, publiés ou non, émanant des établissements d'enseignement et de recherche français ou étrangers, des laboratoires publics ou privés. 


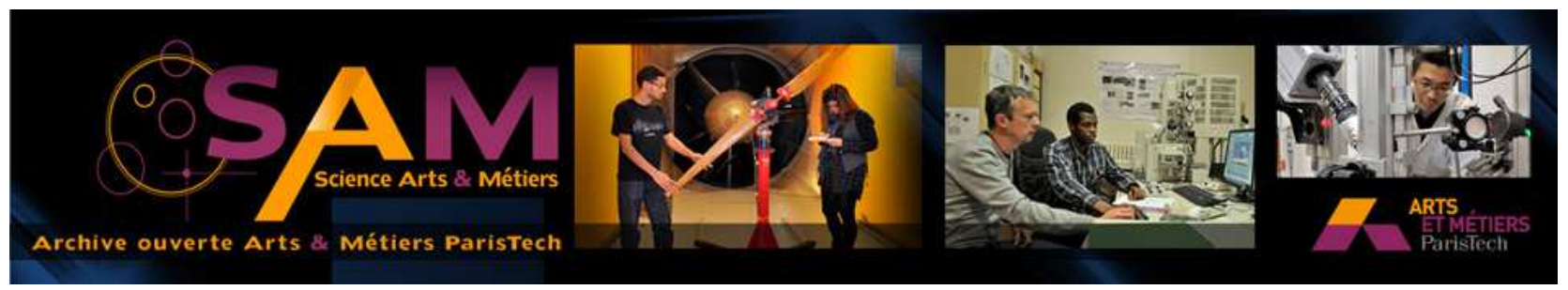

\section{Science Arts \& Métiers (SAM)}

is an open access repository that collects the work of Arts et Métiers ParisTech researchers and makes it freely available over the web where possible.

This is an author-deposited version published in: http://sam.ensam.eu

Handle ID: .http://hdl.handle.net/10985/8059

\section{To cite this version :}

Joseph FITOUSSI, Michel BOCQUET, Fodil MERAGHNI - Effect of the matrix behavior on the damage of ethylene-propylene glass fiber reinforced composite subjected to high strain rate tension - Composites : Part B - Vol. 45, p.1181-1191- 2012 


\title{
Effect of the matrix behavior on the damage of ethylene-propylene glass fiber reinforced composite subjected to high strain rate tension
}

\author{
Joseph Fitoussi ${ }^{\text {a }}$, Michel Bocquet ${ }^{a}$, Fodil Meraghni ${ }^{\text {b, }}$ \\ ${ }^{a}$ Arts et Métiers ParisTech, PIMM - UMR CNRS 8006, 151 Boulevard de L'Hôpital, Paris 75013, France \\ ${ }^{\mathrm{b}}$ Arts et Métiers ParisTech, LEM3 - UMR CNRS 7239, 4 rue Augustin Fresnel, Metz Technopôle 57070, France
}

Keywords:

A. Polymer-matrix composites (PMCs)

B. Debonding

B. Interface/interphase

C. Micro-mechanics

Damage mechanisms

\begin{abstract}
a b s t r a c t
This study investigates the origin of the strain rate effect on the mechanical behavior of a discontinuous glass fiber reinforced ethylene-propylene copolymer (EPC) matrix composite. This kind of composite materials are commonly used for automotive functional and structural applications. To this aim, a multi-scale experimental approach is developed. The deformation processes and the damage mechanisms observed at the microscopic scale are related to the material mechanical properties at the macroscopic scale. Tensile tests up to failure and specific interrupted tensile tests have been optimized and performed for high strain rates up to $200 \mathrm{~s}^{-1}$ to quantify the strain rate effect at different scales. High speed tensile tests have also been performed on the pure copolymer matrix. The threshold and the kinetic of damage have been quantified at both microscopic and macroscopic scales. Experimental results show that the composite behavior is strongly strain-rate dependent. The multi-scale analysis leads to the conclusion that the strain rate effect on the damage behavior of the EPC matrix composite is mainly due to the viscous behavior of the EPC matrix. SEM observations and analysis show that a localized deformation in the interface zone around fibers occurs at high strain rates and directly affects the visco-damage behavior. It is established that when the strain rate increases, the local deformation zone around the fibers behaves like a dissipation zone. Consequently, the damage initiation is delayed and the related kinetic is reduced with respect to the quasi-static loading case.
\end{abstract}

\section{Introduction}

Glass fiber reinforced polypropylene polymers and copolymers composites are very attractive due to their potential for impressive strength-to-weight ratios and impact energy absorption. They have become increasingly employed in automotive and energy applications. Thermoplastic matrix composites offer many advantages compared to thermosetting polymer matrix composites such as low cost, rapid production and capability of complex geometry through an injection molding process [1-4].

More specifically, polypropylene copolymers or rubber modified polypropylene (PP) are able to resist to extreme external conditions such as impact or fatigue loadings. Since 1970, PP or PP copolymers (PPC) have been employed for automotive applications in various components such as bumpers, front, back and side protections and spoilers. Since the 1990s, the reinforcement of PP or ethylene-propylene copolymer (EPC) with discontinuous glass fibers was of high interest for automotive applications because of their low price, lightweight and interesting specific properties.

\footnotetext{
$\Uparrow$ Corresponding author. Tel.: +33 387375459 .

E-mail address: fodil.meraghni@ensam.eu (F. Meraghni).
}

Their application was initially restricted to some semi-structural components such as door panels [5].

However, significant progress in the manufacturing process confers to these composites a good aptitude for injection molding even in the case of complex geometry. Consequently, structural applications are emerging, such as vehicle front end and crash structures [6,7].

Currently, these composite materials as employed for structural components in several fields and especially in the automotive industry [8]. However, their impact resistance during a crash is one of the major concerns related to the passengers' safety. Indeed, these composite materials can be subjected to high strain rate loading during a moderate impact or crash [9]. The amount of absorbed energy during a collision must be sufficient to ensure more reliable residual stiffness and strength. Thus, to optimize the structures crashworthiness, it becomes imperative to study the dynamic behavior of the discontinuous reinforced polypropylene matrix composites. In fact, the strain rate dependent stress-strain curves are the required input for dynamic finite element analysis devoted to the crashworthiness and post-impact behavior prediction. Therefore, it is important to understand the origin of the strain rate effects in these materials. Multiple mechanisms of degradation and 
energy absorption are activated during an impact event both macroscopically [10] and microscopically [11,12].

The strain-rate effects have been investigated on in-plane shear behavior of glass/epoxy unidirectional composites [13,14]. A comparison of the strain-rate sensitivity and the impact response between unidirectional and woven composites has been recently published in [15]. Dasari et al. [16] investigated by scanning electron microscope (SEM) the microstructural evolution of high and low crystallinity PP and ethylene-propylene copolymers during tensile deformation at various strain rates. They depicted the different modes of deformation in the form of strain rate-strain diagrams in which, for a given strain rate, the sequence of the occurring mechanisms can be read until the final rupture of the specimen. Depending on the strain rate, several mechanisms can be observed: crazing, deformation bands, ductile plowing, wedge formation and tearing and finally brittle fracture.

In a recent study, Jerabek et al. [17] performed volume strain measurement and acoustic emission investigation to determine the micro-mechanisms of deformation taking place during tensile tests carried out on particulate filled PP composites for different temperature and strain rates. They highlighted a modification in the dominant micro-mechanism that depends on the strain rate and the temperature. This can occur as a local cavitation mechanism and particle debonding for high strain rate and room temperature, or as a more significant contribution of local shear yielding for low strain rate or high temperature.

Sarang and Misra [18] studied the evolution of the micro-mechanisms of deformation and plastic deformation of an ethylene-propylene copolymer pure or reinforced by wollastonite with regard to strain sensitivity. These authors also show a modification of these mechanisms as a function of the strain rate.

To understand the origin of the observed visco-damage effect, a recent study [19] develops a three-dimensional finite element model using periodic boundary conditions on a representative volume element. The authors adopted strain rate dependent behavior laws which also integrate several damage mechanisms effects. They utilized an explicit formulation in the ABAQUS software [20] to evaluate the influence of the microstructural morphology on micromechanical the stress-wave propagation in the case of high strain rate. The authors also showed an evolution of mechanism as a function of the strain rate, microstructure and loading conditions.

Glass fiber reinforced polymers generally exhibit a diffuse (scattered) damage during loading thus leading to very good properties of energy absorption during impact and crash. However, the damage threshold and the kinetic are widely sensitive to the strain rate effect $[11,12,21]$. An experimental methodology devoted to investigate strain-rate effects on overall composite behavior for moderate rates (up to $200 \mathrm{~s}^{-1}$ ) and based on interrupted dynamic tests has been developed and optimized [11]. This experimental methodology is applied in this work. It has helped to emphasize the strain rate effects on the microscopic and macroscopic behavior of SMC-R26, carbon/epoxy woven laminate composites and glasspolyester long fiber composites [21]. As the strain rate is increased, noticeable effects consist of a delayed damage onset followed by a slightly reduced damage accumulation kinetic. It was established that the strain rate brings about a viscous nature of damage evolution thus leading to the notion of the visco-damage behavior, which characterizes both tested composite materials [11,12, 21,22].

In a relevant recent work by Reiss et al. [23], it has been reported that the glass-fiber reinforced polymer (GFRP) laminates exhibited strain rate and temperature sensitivities. The authors analyzed the coupled effects of strain rate and temperature under tensile tests and showed that they affect the ultimate strengths and the elastic modulus. They give rise to an elasto-viscoplastic behavior of the GFRP. As shown in the literature cited above, glass fiber reinforced thermoplastic such as ethylene-propylene can also develop a nonlinear viscoelastic or viscoplastic behavior. Deformation and damage occur at the microscopic scale and have a large consequence on the macroscopic response. In the literature, the increase of the overall properties of reinforced thermoplastic composites observed for higher strain-rate is described as positive strain-rate dependence [24]. Several other authors studied the effect of the strain-rate coupled to the temperature for short glass fiber reinforced polybutylene terephthalate [25]. It is also important to investigate the effect of the strain rate on the microscopic deformation and damage mechanisms to relate them to the macroscopic dynamic overall response.

After describing the material microstructure, this paper aims firstly at quantifying the effect of the strain rate (from quasi-static to $200 \mathrm{~s}^{-1}$ ) on the overall response of EPC matrix composite at the macroscopic scale in terms of longitudinal Young's modulus, damage threshold and ultimate properties. Secondly, it aims at identifying the predominant mechanisms of deformation and damage occurring at the microscopic scale using a multi-scale analysis coupled with interrupted high speed tensile tests and SEM observations. The effect of the viscous behavior of the EPC matrix on the fiber-matrix interface damage evolution is analyzed and related to the overall properties of the composite. Finally, the strain rate effect on the threshold and kinetic of damage at both macroscopic and microscopic scales is quantified and discussed.

\section{Microstructure and material specification}

The studied material is obtained by injection molding and consists of an ethylene-propylene copolymer matrix reinforced by discontinuous glass fiber. The average diameter of the glass fibers is approximately $16 \mathrm{~lm}$ (Fig. 1) and the average length is up to $1.2 \mathrm{~mm}$. The composite material is presented in the form of $3.2 \mathrm{~mm}$ thickness plates. The plates are extracted from specific planar zones of actual structural automotive parts.

The weight content of the glass fibers is determined using the calcination technique $\left(500{ }^{\circ} \mathrm{C}, 5 \mathrm{~h}\right)$, which is carried-out according to EN ISO 1172 standard. The fiber weight content is measured on nine composite samples extracted from different locations into an automotive component. The average value is $39 \pm 0.6 \mathrm{wt} . \%$ and corresponds to a fiber volume fraction up to $19.7 \%$.

The orientation distribution of the fibers in the thermoplastic matrix has been investigated by image analysis on SEM micrographs performed upon a representative element volume whose the observed surface was $2.5 \times 3.2 \mathrm{~mm}^{2}$. The results shown in Fig. $2 b$ represent the distribution of the fiber content as a function of the orientation angles $\mathrm{u}$ (in-plane angle) and h (out-of plane angle) which are defined in Fig. 2a. Thus, to some extent, the experimental findings lead to several partial conclusions:

- The majority of the fibers (more than $60 \%$ ) is contained in the plane of the plate $(X-Y)$ and are accordingly characterized by an angle $\mathrm{u}=90^{\circ}$. These fibers are oriented according to the Mold Flow Direction (MFD) corresponding to $\mathrm{h}=0^{\circ}$.

- About $10 \%$ of the fibers are oriented at $\mathrm{h}= \pm 45^{\circ}$ and are inclined out-off plane $(X-Y)$. Their angles are around $\mathrm{u}= \pm 45^{\circ}$.

- A few of the fibers (roughly 5\%) are oriented perpendicular to the MFD $\left(\mathrm{h}=90^{\circ}\right)$. Nevertheless, this small fraction of disoriented fibers remains contained in the plane of the plate $\left(\mathrm{u}=90^{\circ}\right)$.

Thus, it can be assumed that due to the injection molding process, most of the fibers remain into the plane and are oriented parallel to the Mold Flow Direction (MFD). This confers an overall 


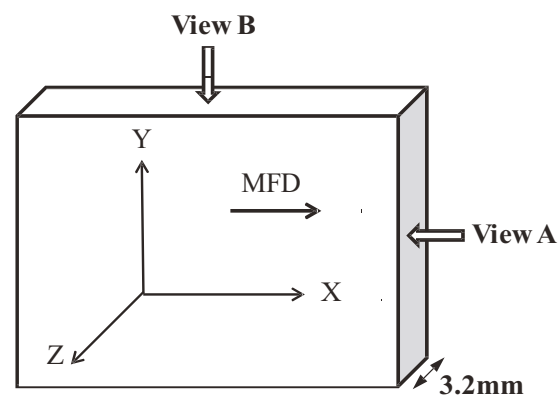

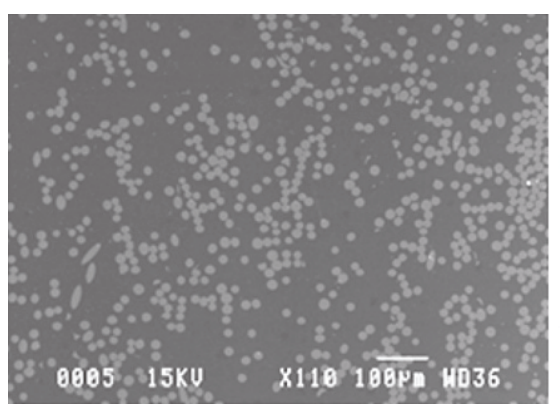

View A

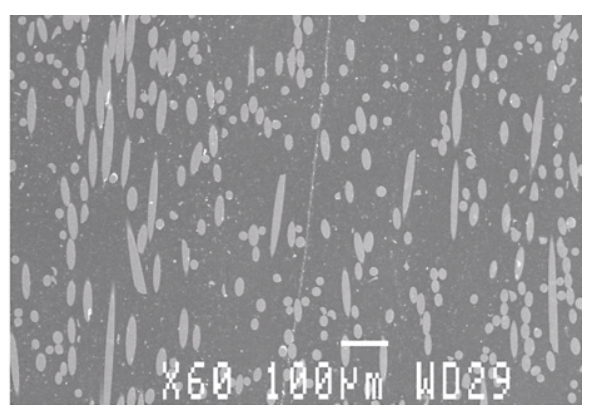

View B

Fig. 1. SEM observations of the microstructure according to two view planes of the composite ethylene-propylene copolymer matrix reinforced by discontinuous glass fibers.

(a)

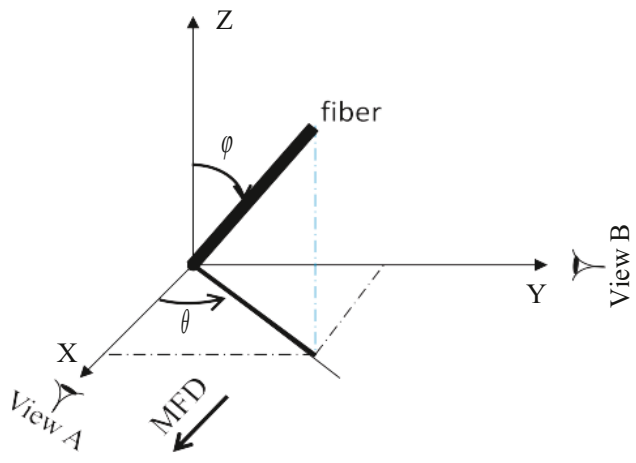

(b)

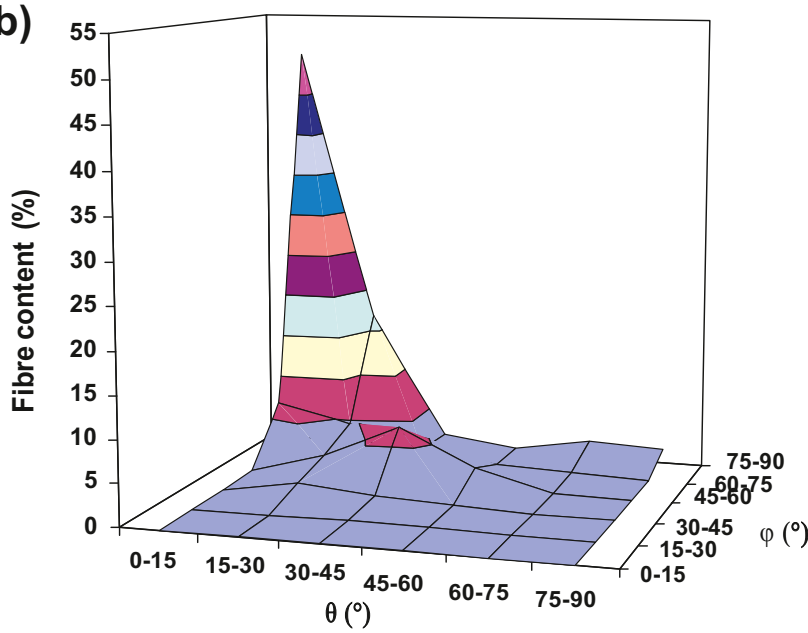

Fig. 2. (a) Definition of the fiber orientation angles. (b) Fiber orientation distribution measured by image analysis.

orthotropic behavior for the material, which can be characterized by a longitudinal direction $(X)$ parallel to the MFD and a transversal direction $(Y)$. In this work, the high speed tensile tests, the experimental investigation and the multi-scale analysis of the strain rate effect are limited to the longitudinal direction.

\section{Experimental approach and testing devices}

In the present study, high-speed tensile tests have been conducted upon a servo-hydraulic machine (Schenck) which is equipped with a launching system. It can reach a crosshead speed range from $10^{-4} \mathrm{~m} / \mathrm{s}$ to $20 \mathrm{~m} / \mathrm{s}$. The dynamic load is measured by using a piezo-electric crystal load cell having a capacity of $50 \mathrm{kN}$.

The developed experimental approach is widely detailed elsewhere [11]. It enables the experimental characterization of the composite material for strain rate ranging from quasi-static to dynamic solicitation $\left(200 \mathrm{~s}^{-1}\right)$. The experimental methodology devoted to high-speed tensile tests has been developed by optimizing two main aspects: damping joint and specimen geometry. The experimental approach indeed combines experimental and numerical analysis aimed at optimizing specific sample dimensions and a damping joint which enables minimizing the perturbation due to the propagation of dynamic shock waves as detailed in [11].

The first aspect dealt with the damping joint. Indeed, the transient dynamic loading nature coupled to the testing system inertial effects produce a dynamic shock wave whose magnitudes have to be minimized. The damping joint inserted between the sliding bar and the tube of the hydraulic jack enables a partial absorption of the generated stress wave. It attenuates the shock wave during its compression. Note that the compression of the damping joint should be completely finished before the end of the total elastic deformation of the specimen. This corresponds to a rise time ranging from $10^{-4} \mathrm{~s}$ to $10^{-6} \mathrm{~s}$ and depends on the adopted joint thickness and the nominal test velocity.

The optimization has led to the selection (constitutive material) and the optimization (thickness) of a damping joint. It consists of a low impedance material such as a rubber nitrile and its thickness varies from $1.5 \mathrm{~mm}$ to $3 \mathrm{~mm}$ as function of the expected strain rate level. 


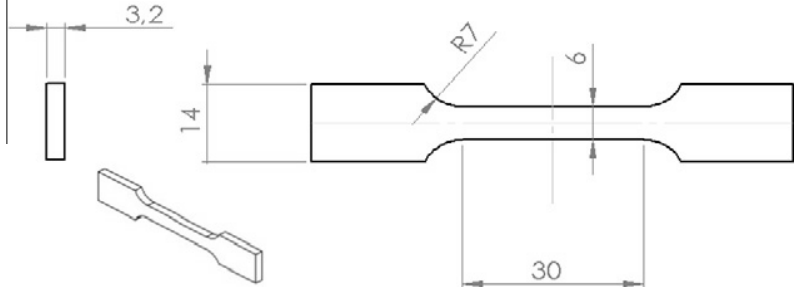

Fig. 3. Specimen geometry optimized for high speed tensile tests of short glass fiber reinforced polymer matrix composites.

The second aspect of the experimental methodology development dealt with the specimen geometry optimization. The specimen has dumbbell-shaped geometry and the optimization procedure was achieved by numerical computation using the ABAQUS finite element code to determine the optimal dimensions, namely: the width of the narrow portion, the length of the narrow parallel-sided portion and the blending radius. The initial dumbbell-shaped geometry was inspired by the ISO 37 type 1 (ASTM D 638 ) standard. The optimization criterion consisted of reaching a stabilized stress/strain distribution within the specimen narrow parallel-sided portion. Fig. 5 given in the reference [11], showed the spatio-temporal contour plot of the longitudinal stress calculated along the central line of a specimen made of SMC composite and subjected to a dynamic loading at an imposed velocity of $1 \mathrm{~m} /$ $\mathrm{s}$. It was showed that from the time increment of $3.5 \mathrm{E}-5 \mathrm{~s}$ the stress is stabilized in the narrow parallel-sided portion. Thereby, the recursive FE-optimization procedure resulted in the determination of optimal dimensions.

For the glass-fiber reinforced EPC matrix composite, the dumbbell-shaped specimen geometry and its optimal dimensions are similar to that optimized for the SMC and are given in Fig. 3. The strain is measured using strain gages positioned at the central zone of the specimen as well as by means of a laser extensometer measuring the relative displacement of two points located at the edges of the sample's central zone. Note that the resolution of the laser extensometer is $401 \mathrm{~m}$. The sampling frequency of the acquisition system utilized for the laser extensometer and for the strain gages can be set from $1 \mathrm{MHz}$ to $50 \mathrm{MHz}$. It depends on the nominal test velocity. The strain rate is then determined directly by a temporal derivation of these strain measurements. It has been established that both measurements give similar results [26].

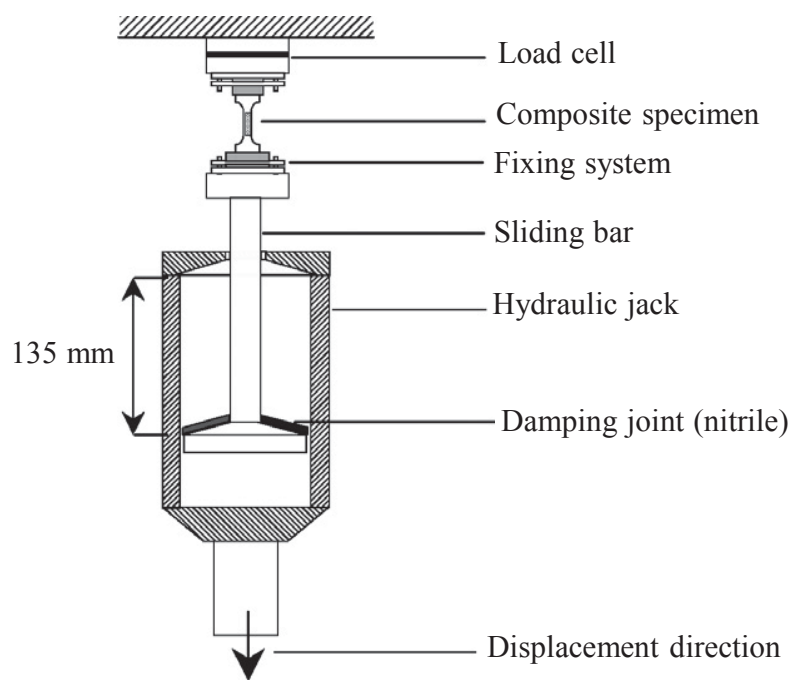

Fig. 4. Servo-hydraulic testing device utilized for high-speed tensile tests until specimen rupture.

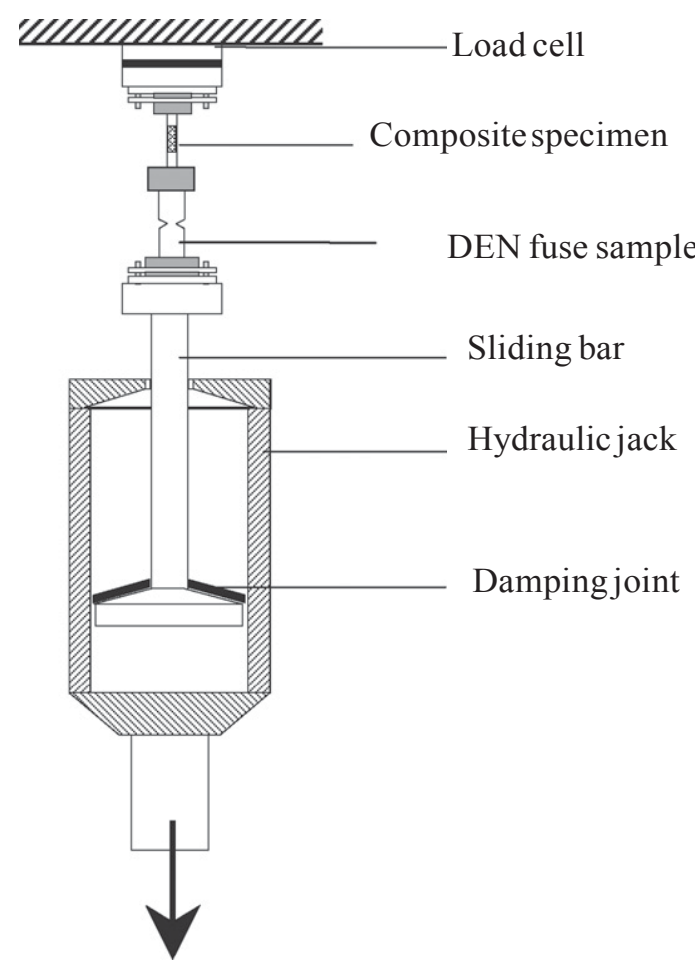

Fig. 5. Schematic diagram of the servo-hydraulic testing device utilized for interrupted high-speed tensile tests.

High speed tensile tests were carried out at different strain rates according to two experimental procedures.

\subsection{High-speed tensile tests until rupture}

The first set of high-strain rate tensile tests has been performed at different strain-rates until the composite specimen total failure. The composite specimen is placed between the load cell (upper extremity) and the moving device (lower extremity) as schematized in Fig. 4. Prior to the contact between the sliding bar and the hydraulic jack, the latter one is accelerated over a straight displacement of $135 \mathrm{~mm}$ in order to reach the nominal crosshead velocity before the load begins. Once the contact occurs, the specimen is then subjected to a tension at a constant load-rate. The optimized damping joint placed between the slide and the hydraulic jack attenuates partially the wave effects produced by the $\mathrm{dy}^{-}$ namic shock.

\subsection{Interrupted high-speed tensile tests}

The interrupted tensile test technique has been conducted in order to quantify the damage effect as a function of the applied strain rate. This experimental methodology consists of interrupting the high speed loading at fixed stress levels before the total failure [11]. Indeed, the presence of inertial effects during a rapid tensile test makes it difficult to interrupt the high speed loading before the total failure of the specimen. An original solution consists in loading the specimen simultaneously with a double-edge notched (DEN) fuse sample (Fig. 5) characterized by brittle fracture. The fuse ligament width is associated with the suitable failure load level to be reached when the test should be interrupted. The interruption of the high speed tensile test thus brings about a stress release in the specimen. This procedure is repeated several times for the same specimen by changing the fuse ligament width before each specimen is reloaded. By doing so, one can reach a load level 
greater than the previous one. The interrupted test methodology thus enables to reach and then to monitor different damage level for the studied material.

4. Strain rate effect on the mechanical properties at the macroscopic scale

One of the aims of the present work is to investigate the origin of the strain rate effect on the mechanical properties of the glass fiber reinforced EPC matrix composites. As discussed in $[14,24,27]$, there is not yet a valid standard for the high-speed testing of reinforced composites. In addition, the main issue of such tests is how to obtain both a homogeneous strain field and a constant strain rate during the dynamic loading. To this end, the experimental methodology previously presented in Section 3 has been applied on the studied composite material. The optimization of the specimen geometry and the use of the damping joint reduced and attenuated the shock wave during its compression in particular for moderate strain rates. They enabled a partial absorption of the generated stress wave and the related oscillations. It should be pointed out that the damping joint would be completely compressed before the end of the total elastic deformation of the specimen. This compression time corresponds to a rise time ranging from $10^{-4} \mathrm{~s}$ to $10^{-6} \mathrm{~s}$ which depends on the adopted joint thickness and the nominal test velocity.

Fig. 6a shows an example of the evolution of the strain measured by the laser extensometer at a moderate strain rate of $15 \mathrm{~s}^{-1}$. For a strain rate of $15 \mathrm{~s}^{-1}$, the strain-time curve shows that the slope corresponding to the strain rate is rapidly stabilized after a rise time of about $1.5 \times 10^{-4} \mathrm{~s}$ when the total testing time is $1.5 \times 10^{-3} \mathrm{~s}$. The strain rate is then estimated on the linear part of the curve.

However, for high strain rates, it is difficult to get a constant strain rate. In fact, the inertial effects may require a thicker damping joint. Consequently, the damping stage is relatively longer with regard to the total time of the test. Thereby, the strain rate measured on the specimen becomes really constant relatively later. Fig. $6 \mathrm{~b}$ shows the evolution of the strain rate during a high speed test. The average strain rate is approximately $208 \mathrm{~s}^{-1}$ and the corresponding rise time is $6 \times 10^{-5} \mathrm{~s}$ whereas the total testing time is $2 \times 10^{-4} \mathrm{~s}$.

\subsection{Typical stress-strain curves from dynamic tensile tests until failure}

High speed tensile tests have been carried out up to failure at different strain rates. Regardless of strain rate, the composite material exhibits a nonlinear response characterized by the first non-linearity which begins at a stress level corresponding to $30 \%$

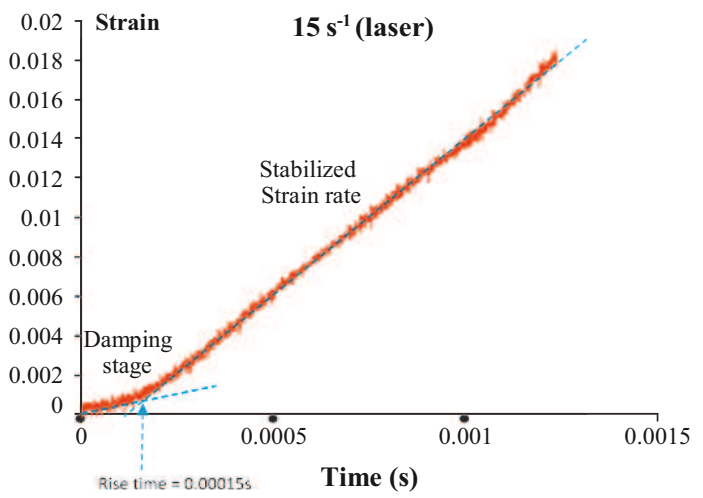

Fig. 6. (a) Measured strain versus time curve for a moderate speed tension test бe $1 / 415 \mathrm{~s}^{-1} \mathrm{P}$. (b). Measured strain versus time curve for a high speed tension test бe $\mathrm{e}_{\text {moy }} 1 / 4208 \mathrm{~s}^{-1} \mathrm{p}$.

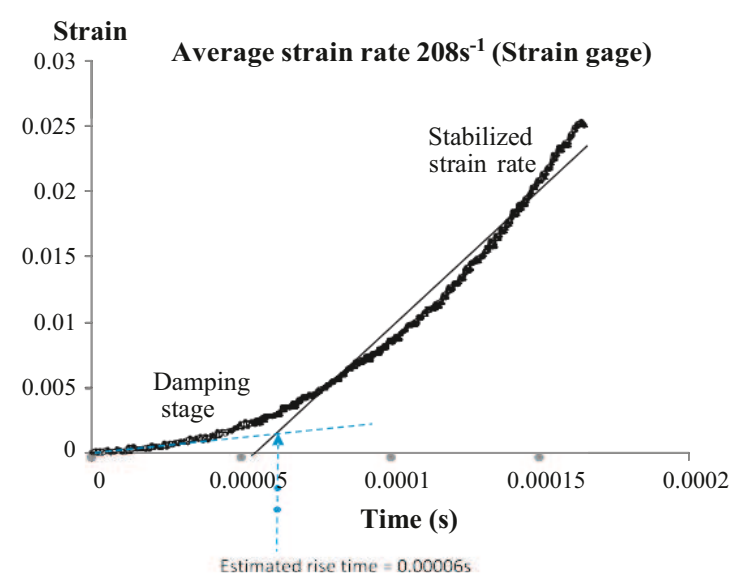

Fig. 6 (continued)

of the maximum stress. Typical tensile curves show three stages, as given in Fig. 7. The first one is linear and corresponds to the elastic behavior. It is followed by a second stage characterized by the socalled "knee point" which corresponds to the beginning of the nonlinear behavior. Basically, this point matches the damage initiation and is associated to the damage threshold. Finally, the third stage exhibits a quasi-linear evolution of the stress as a function of strain until the final failure. Comparatively, for a glass fiber reinforced thermoset matrix composite such as SMC [11,12,23,28] the first part corresponds to the elastic behavior. The second part is related to the onset of a diffuse damage mostly caused by fiber-matrix interface debonding and matrix micro-cracking. Finally, during the third part of the curve, the damage propagates until final failure of the specimen.

The following sections demonstrate through an analysis at the microscopic scale, how the viscous behavior of the EPC matrix phase can affect the damage threshold and kinetic of the glass fiber reinforced EPC matrix composite.

\subsection{Strain rate effect on the overall mechanical properties}

Fig. 8 shows that the overall mechanical behavior of the glass fiber reinforced EPC matrix varies significantly under rapid straining as compared to quasi-static loading. The dynamic behavior of the studied material is widely strain-rate dependent.

The Young's modulus is calculated by linear regression in the linear part of the stress-strain curve. For the lower strain rates,

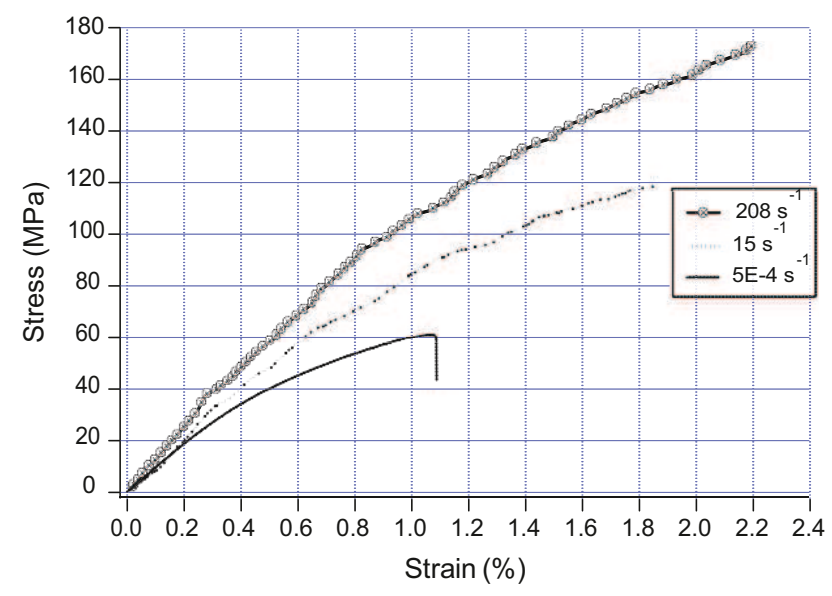

Fig. 7. Typical EPC matrix composite stress-strain tensile curves. The three characteristic stages can be shown for all strain rates. 

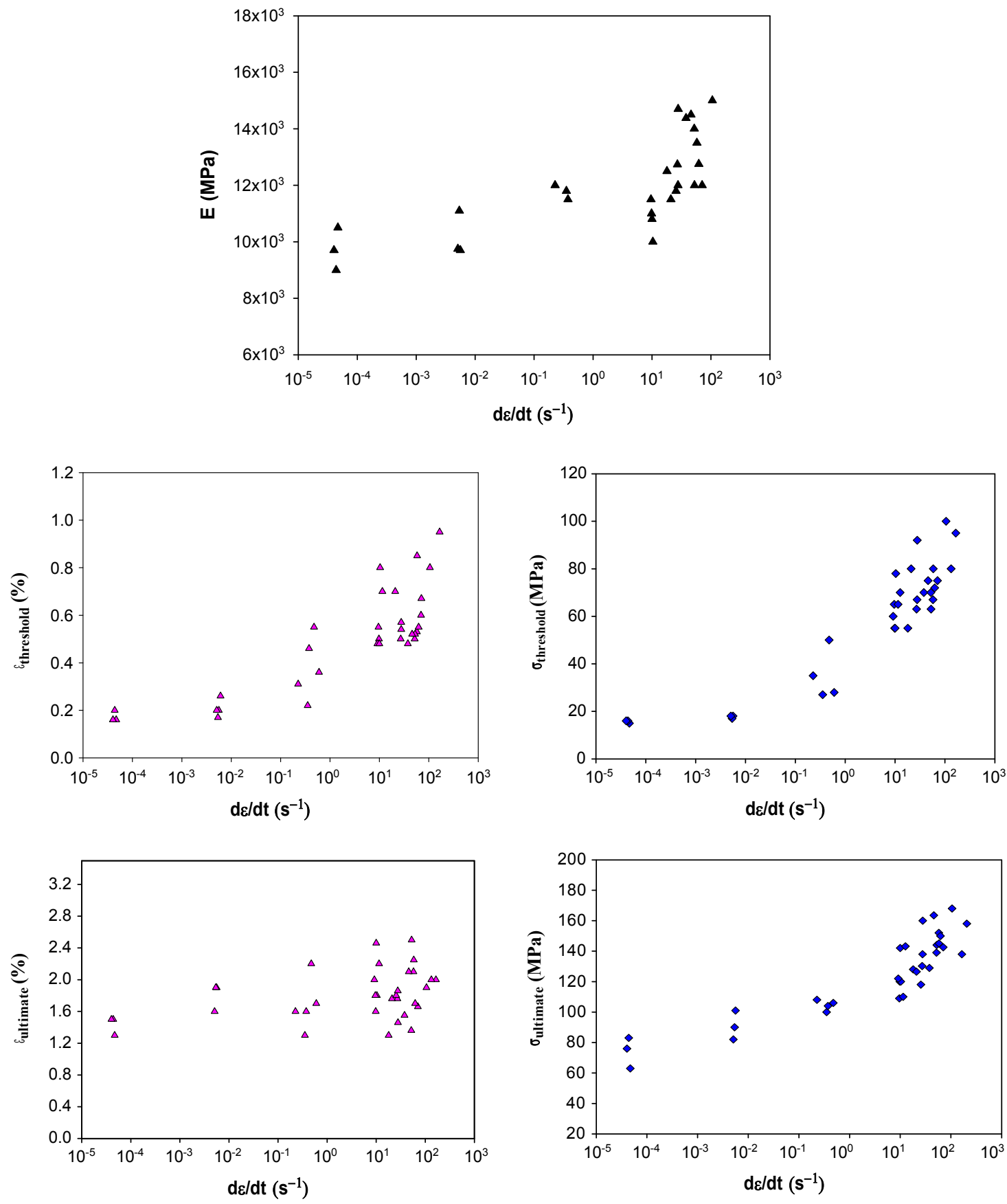

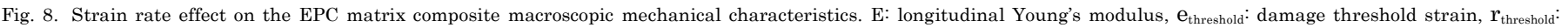
damage threshold stress, $\mathrm{e}_{\text {ultimate }}$ : ultimate strain, $\mathrm{r}_{\text {ultimate }}$ : ultimate stress.

the interval is chosen beyond the damping stage corresponding to the rise time. For higher speed tests, the indicated strain rate corresponds to the average value taken on the linear part of the curve. The Young's modulus is thus extrapolated from the linear part corresponding to the tangent line to the origin.

The obtained scattered experimental results of Fig. 7 are due to the variation of the microstructure induced by the injection process which characterizes typically the short glass fiber reinforced thermoplastics. Indeed, one has to notice that tested specimens in this study were cut from plates which have been extracted from specific planar zones of actual structural automotive parts. In addition, due to the damping stage during the rise time, the strain rate is not exactly constant in particular for high speed tests. The indicated value of strain rate is an average value. Furthermore, one can observe that the scattering increases for high strain rate. The applied experimental methodology assures that the specimen is submitted to a homogeneous macroscopic strain and a relatively constant strain rate. However, at the local scale, as shown in a recent paper [19], the microstructure has a strong effect on the stress-wave propagation at the local scale. Local inertial effect affects the local stress state notably for high speed tests. Microstructure variation may cause different damage evolution even if the specimen seems stabilized at the macroscopic scale. This effect is more noticeable for high strain rate and particularly affects the maximum strain shown see in Fig. 7 .

The analysis of the stress-strain curves obtained in tension for different strain rates ranging from quasi static to $200 \mathrm{~s}^{-1}$ allows plotting the evolution of the longitudinal Young's modulus and 
Table 1

Thermal properties and crystallinity ratio of the EPC matrix and glass fiber reinforced EPC matrix composite.

\begin{tabular}{lllc}
\hline & $\mathrm{V}$ & $T_{f}\left({ }^{\circ} \mathrm{C}\right)$ & $T_{\text {Crys }}(\% \mathrm{C})$ \\
\hline EPC matrix without fiber & 34.5 & 168.9 & 132.5 \\
EPC matrix into composite & 35.0 & 167.6 & 132.4 \\
\hline
\end{tabular}

the damage thresholds in terms of strain $\left(\mathrm{e}_{\text {threshold }}\right)$ and stress $\left(r_{\text {threshold }}\right)$ as functions of the strain rate. The ultimate strain and stress are also shown in Fig. 8. However, the microstructure variability of this class of materials can bring about a discrepancy in particular for dynamic loadings. It can explain the dispersion of the obtained results shownin Fig. 8. The evolution of allinvestigated properties shows clearly that the mechanical behavior of the studied composite is strongly load rate dependent. It has been observed that an increase of the strain rate gives rise to improved overall mechanical characteristics in terms of damage threshold and kinetic. However, it is worth noting that this increase is noticeably more pronounced after a strain rate beyond to $10 \mathrm{~s}^{-1}$. Similar experimental results have been obtained by Schoßig et al. [24] for glassfiber reinforced polypropylene and polybutene- 1 composites. They showed that, for these materials, tensile strength and the appearance of fracture are strongly strain-rate dependent.

In contrast to glass fiber reinforced thermoset matrix composites [11-14,21,22], the elastic properties of the EPC matrix based composites are sensitive to the strain rate effect. For the studied composite material, the Young's modulus increases from $10 \mathrm{GPa}$ for quasi-static tests to $15 \mathrm{GPa}$ when tested at $200 \mathrm{~s}^{-1}$, which corresponds to an increase of $50 \%$. The damage stress threshold increases from $20 \mathrm{MPa}$ under quasi-static loading to more than $100 \mathrm{MPa}$ for dynamic loading (500\% increase). At the same time, the ultimate stress is delayed from $80 \mathrm{MPa}$ to $160 \mathrm{MPa}(100 \%$ increase). The effect of strain rate appears through an increase in the elastic modulus, the damage thresholds and ultimate strain and stress. The ultimate properties and the damage threshold increase significantly at high strain rate. As illustrated in Fig. 8, one can notice that the strain rate effect is more visible and marked for the ultimate stress than for the ultimate strain.

\subsection{Strain rate effect on the pure matrix behavior}

Table 1 shows a comparison of the thermal properties of the composite and those measured on the pure EPC matrix. It summarizes for both materials: $T_{f}$ (melting temperature), $T_{\text {Crys }}$ (temperature of crystallization) and $\mathrm{v}$ (crystallinity ratio). The obtained results are similar for both the unreinforced and reinforced EPC

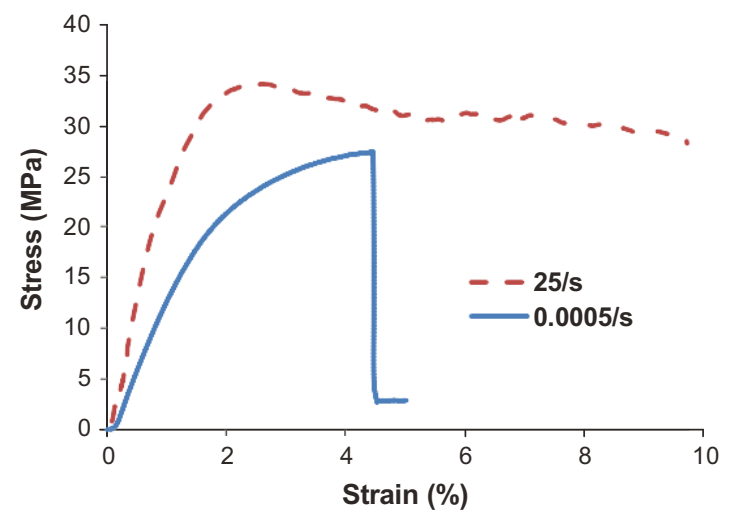

Fig. 9. Typical EPC pure matrix (without reinforcement) stress-strain tensile curves. matrix. It can be stated thus that the thermo-mechanical behavior of the thermoplastic matrix is not affected in a significant way by presence of the reinforcement.

Nevertheless, the mechanical behavior of thermoplastic matrix is also affected by the strain rate. To understand the origin of the strain rate effect presented in the previous section, the effect of strain rate on the pure thermoplastic matrix phase of the composite is analyzed. On the basis of the results given in Table 1, one can notice that the thermal properties governing the thermo-mechanicalbehavior of the pure matrix are similar to those of the composite having the same thermoplastic matrix. Then, it can be reasonably assumed that the strain rate effect measured on the pure matrix should be quite representative, at least qualitatively, of that observed for the composite. Fig. 9 shows the pure matrix tensile behavior for two different strain rates: quasi-static and a moderate strain rate $\left(25 \mathrm{~s}^{-1}\right)$. An important evolution is observed in terms of the matrix Young's modulus, the strain and stress damage thresholds. These are increasing significantly. In particular, for the quasi-static test $\left(5.10^{-4} \mathrm{~s}^{-1}\right)$, longitudinal Young's modulus is about 1.39 GPa whereas for a dynamicloading $\left(25 \mathrm{~s}^{-1}\right)$ it increase to $2.95 \mathrm{GPa}$. Moreover, it should be noticed that the ultimate strain for a quasi-static loading is about $4.5 \%$ but can reach $10 \%$ for a dynamic loading at $25 \mathrm{~s}^{-1}$. So, a high strain rate leads to a significant increase in matrix deformation. This aspect is important to understand the inelastic deformation mechanisms of the composite when submitted to high strain rates.

\section{Multi-scale analysis of the strain rate effect on damage}

Experimental results show that the tensile strength of the studied composite material increases noticeably with the increase of strain rate. This is mainly due to a shift of the first non-linearity corresponding to the appearance of a non-elastic or non-linear physical phenomenon occurring at the microscopic scale. In the case of thermoset resin reinforced composites, it has been shown than the principal mechanism for the shift of the non-linearity was the fiber-matrix interface debonding [11,12,21,26,28]. In the case of thermoplastic reinforced composites, the rheology of the matrix, through viscoelasticity or viscoplasticity, governs the non-linear behavior of the composite. To evaluate the influence of the damage mechanisms and the rheology of matrix, the interrupted test method is applied. This method has been previously optimized and allowed to:

- Describe the evolution of the stiffness reduction of the composite during dynamic loading.

- Investigate the evolution of the micro-mechanisms at the local scale.

- Understand and to quantify the influence of the micro-mechanisms on the overall properties of the composite.

In the following section, the results obtained from interrupted test are described in terms of damage analysis at the macroscopic and the microscopic scales of material.

\subsection{Strain rate effect on damage at the macroscopic scale}

To evaluate the rate dependence of the damage in terms of initiation and growth, the damage analysis was conducted at both the macroscopic and the microscopic scale. Macroscopic damage can be quantified by tensile modulus reduction using the well-known continuum damage mechanics. The damage scalar variable $\left(D_{\text {macro }}\right)$ is thus expressed [29]:

$D_{\text {macro }} 1 / 41-\frac{E^{D}}{E^{0}}$ 
(a)

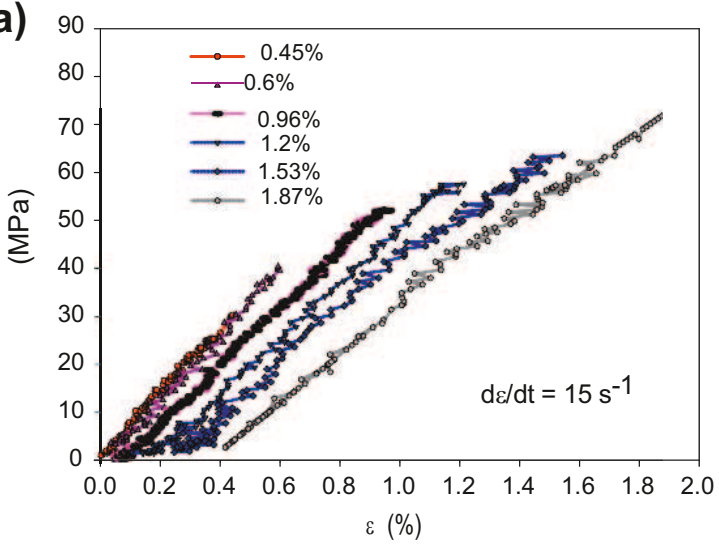

(b)

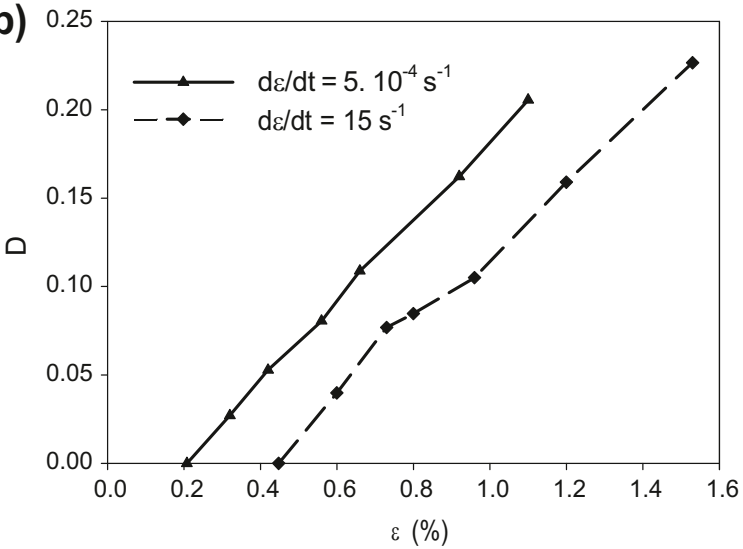

Fig. 10. (a) Loading-unloading stress-strain curves (dynamic case). (b) Macroscopic damage parameter evolution for both quasi-static and dynamic cases.

$E^{0}$ and $E^{D}$, are, respectively, the longitudinal Young's modulus of virgin and damaged material. $E^{0}$ is determined by the initial slope of the stress-strain curve. The residual (current) modulus $\left(E^{D}\right)$ of the damaged material is estimated by the slope of the reloading curves after each tensile test interruption at a predefined load level (Fig. 10).

Interrupted high-speed tensile tests have been carried-out for two strain rates: $0.0005 \mathrm{~s}^{-1}$ and $15 \mathrm{~s}^{-1}$. For each "unloading/reloading" loop (Fig. 10a), the evolution of the macroscopic damage parameter $\left(D_{\text {macro }}\right)$ is experimentally estimated and plotted as a function of the strain level reached for both strain rates as shown in Fig. 10b. Experimental results show that increasing the strain rate leads to a delayed damage initiation. Indeed, for the quasi-static loading, the stiffness reduction begins for strain level of $0.2 \%$. For the dynamic loading however the damage first appears at strain of $0.45 \%$. As shown in Fig. 10b, it can be established that the stiffness reduction is rate dependent. Nevertheless, the slopes of the curves of $D_{\text {macro }}$ as a function of the strain show that the damage kinetic is relatively insensitive to the strain rate.

It must be mentioned that micro cracks closure could occur when unloading and essentially for low applied stress/strain levels. However, as shown in Fig. 11, micro cracks do not close completely notably at the fiber/matrix interface even after unloading. The inelastic (permanent) strain measured after unloading is caused by the subsequent crack opening.

\subsection{Damage analysis at the microscopic scale}

This section aims at establishing a relationship between the micro-defects density and the macroscopic behavior in terms of strain/stress curves and the evolution of the scalar damage variable $\left(D_{\text {macro }}\right)$ defined through the longitudinal young's modulus reduction. To this end, a local damage indicator $\left(d_{\text {micro }}\right)$ is proposed to quantify the damage accumulation at the microscopic scale. Several works $[11,12]$ focusing on damage in polymer reinforced fiber composites have been conducted using different experimental techniques such as acoustic emission [30], micrographs and C-Scan [31]. These works point-out that short fiber reinforced composite materials fail according to micro-damage mechanisms such as matrix microcracking, fiber-matrix interface debonding and fiber breakage. These studies have extensively shown that the main sources of damage is firstly the fiber-matrix interface debonding which can appear early ( $20 \%$ of the failure stress) and secondly the matrix microcracking. It is worth mentioning that the same phenomenology is observed for the studied thermoplastic reinforced composite for both quasi-static and dynamic loading: namely that the damage growth is mainly governed by the fiber-matrix

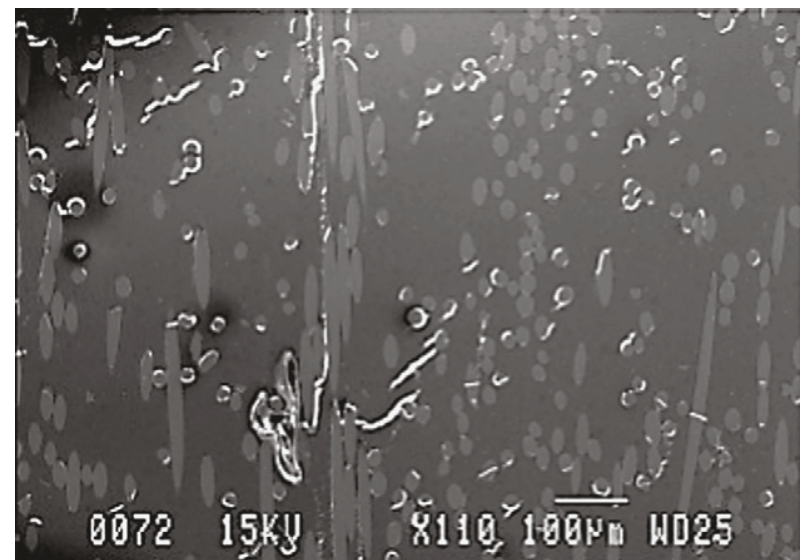

Fig. 11. Microscopic observation of the diffuse damage of glass fiber reinforced EPC under quasi-static load. Damage state observed for a strain level of $\mathrm{e}=1.15 \%$.

interface failure. Consequently, the local damage can be measured through the evolution of the interface failure ratio which constitutes a relevant damage indicator. After each interrupted tensile test, damage accumulation is investigated by means of SEM micrographs performed upon a representative volume element (RVE).

The microscopic damage state is represented at the local scale as follows:

$d_{\text {micro }} 1 / 41-\frac{f_{d}}{f_{v}}$

where $f_{d}$ is the volume fraction of debonded fibers and $f_{v}$ is the fiber volume content in the RVE.

The microscopic investigation has been conducted in order to relate the micro-defects density evolution to the macroscopic stiffness reduction described above. Before each re-loading stage, a microscopic image analysis is achieved by means of SEM micrographs performed on a representative volume element (RVE): the observed surface was of $2.5 \times 3.2 \mathrm{~mm}^{2}$. This means that the investigation zone is large enough to contain all the heterogeneities of the material microstructure. The local investigation can be assumed as statistically representative of the damage accumulation in the studied material composite.

\subsubsection{Quasi-static loading}

Under quasi-static loading, damage mainly begins simultaneously with the first non-linearity on the stress-strain curve $\left(\mathrm{e}_{\text {threshold }}=0.18 \%\right)$. It appears first as fiber-matrix interface failure 


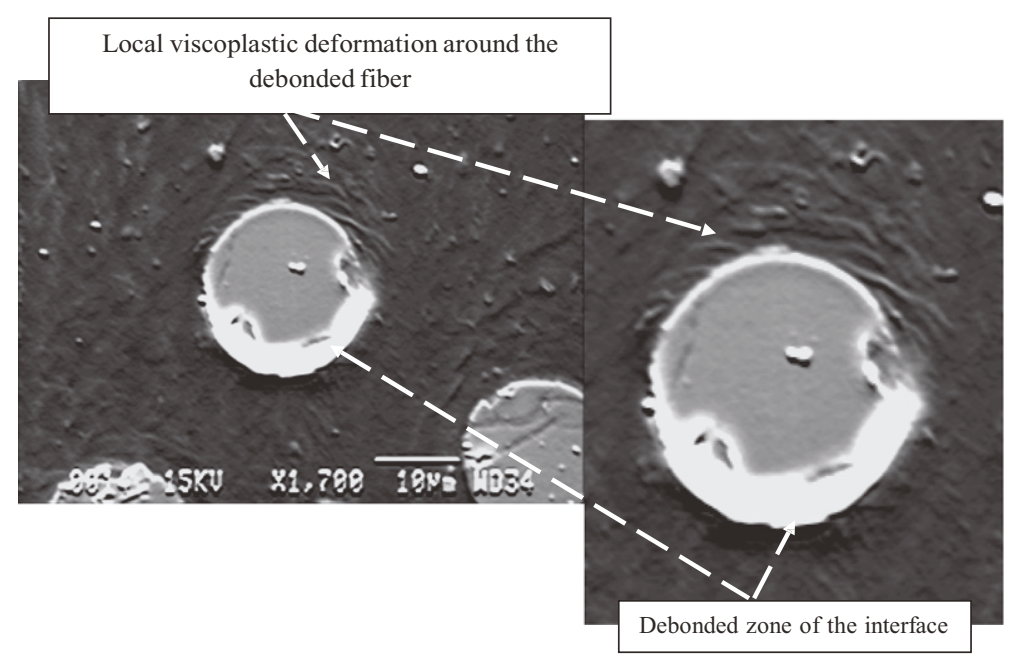

Fig. 12. Matrix local viscoplastic deformation around a debonded fiber.

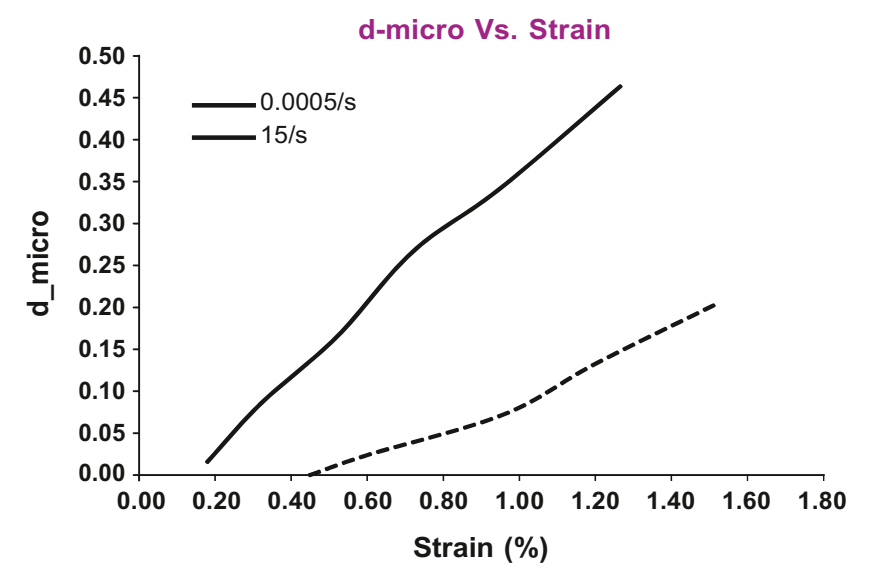

Fig. 13. Comparison between dynamic and quasi-static damage kinetic at the microscopic scale for all fiber orientations considered as an overall microscopic damage indicator.

which occurs on the more disoriented fibers $\left(\mathrm{h}=90^{\circ}\right)$. Then, it progressively propagates to the less disoriented fibers. Besides the interface debonding, one can observe matrix micro-cracking around the debonded fibers. For a strain of $0.4 \%$ which corresponds to the knee point on the stress-strain curve, it is worth mentioning that the fiber-matrix interface failure is generalized on all fiber orientations on the whole volume of the specimen. A diffuse (scattered) damage over the whole investigated zone (Fig. 11) is observed until the final stages of the specimen behavior which occurs after the coalescence of the fiber-matrix interface debonding and the matrix micro-cracks.

\subsubsection{Dynamic loading}

When the material undergoes a high strain rate, no damage is observed before the strain threshold of $0.45 \%$. For the quasi-static case, the first interfacial damage occurs mainly for the $90^{\circ}$ oriented fibers with regard to the maximum stress direction. It then propagates progressively over the whole specimen for all reinforcement fiber orientations. More specifically, for high strain rate, a large influence of the matrix viscosity (rheology) on the damage evolution is observed. In contrast to the quasi-static loading, no matrix micro-cracking is observed around the debonded interfaces. Furthermore, as reported in Fig. 12, onecan observe that the matrix

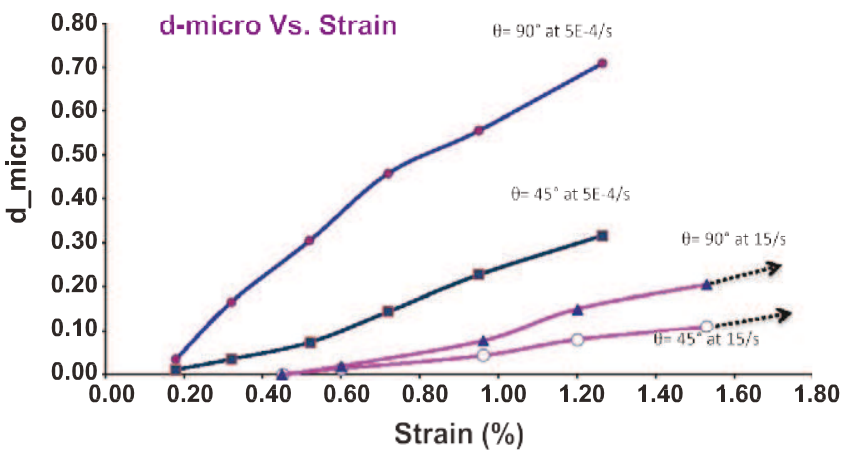

Fig. 14. Quasi-static and dynamic evolution of the microscopic damage indicator with respect to two specific in-plane orientations of fibers: $h=90^{\circ}$ and $h=45^{\circ}$.

is highly strained locally around the fibers. This yields clearly to the statement that the strained zone around the debonded interface dissipates the strain energy and consequently inhibits the interfacial crack propagation through the matrix. Moreover, as discussed above, one can see in Fig. 8 that a large matrix macroscopic strain is reached for high strain rate. This large strain level dissipates the energy and hence contributes to the absence of the matrix micro-cracking mechanism (onset and propagation) for high strain rates. Consequently, the damage occurs on other fibers by localized interface debonding. This leads to a noticeable decrease of the damage kinetic due to the reduction of the micro-cracks propagation and accumulation. Fig. 13 shows a comparison between the quasi-static and the dynamic measured fiber-matrix interface failure kinetic. It should be noticed that the delayed damage threshold accounts for the high stress corresponding to the appearance of nonlinearity observed on the macroscopic stress-strain curve in Fig. 7.

For a strain rate of $15 \mathrm{~s}^{-1}$, the damage occurs firstly through the interfacial decohesion and the related deformation zone. With the increase of the strain, beyond a strain of $1.53 \%$ the interfacial decohesion starts to propagate through the matrix from one fiber to its closer neighbor. These microscopic damage mechanisms intensify through the accumulation of micro-defects leading to macroscopic failure. Moreover, Fig. 14 shows the strain rate effect on the interface debonding for two fiber orientations: $\mathrm{h}=90^{\circ}$ and $\mathrm{h}=45^{\circ}$. For the orientation orthogonal to the maximum stress direction $\left(\mathrm{h}=90^{\circ}\right.$ ), one can evaluate the strain rate effect due to a pure normal stress at the fiber interface while the curves obtained for $\mathrm{h}=45^{\circ}$ also 
shows the strain rate effect due to shear stress which are assumed to be tangential to the interface. Therefore, it is obvious that fibers oriented according to the MDF do not exhibit significant interface debonding for quasi-static and dynamic loading. It can be emphasized that for both studied strain rates, the damage is mainly governed by interface debonding occurring for fibers disoriented with respect to the MFD, namely from $45^{\circ}$ to $90^{\circ}$. Furthermore, as shown in Figs. 13 and 14, the microdamage level and its kinetic decrease when the strain rate increases. In addition, Fig. 14 shows that the more disoriented fibers (with respect to the MFD) predominantly undergo interface debonding. These fibers represent only $20 \%$ of the fiber content. Therefore, their contribution to the longitudinal stiffness is limited. These results lead to the conclusion that, to investigate the degradation kinetic, the micro damage indicator is more relevant than the macroscopic damage variable expressed through the stiffness reduction. Indeed, as shown in Fig. 10b, for a strain rate of $15 \mathrm{~s}^{-1}$, the evolution of the longitudinal Young's modulus is not sensitive to the damage occurring at disoriented fibers due to its low value (Figs. 13 and 14). That can explain why the studied composite material exhibits similar macroscopic damage kinetic for quasi-static and dynamic loading (Fig. 10b).

\section{Conclusion}

This paper proposes a multi-scale analysis of the mechanical behavior of a short fiber reinforced ethylene-propylene copolymer (EPC) matrix composite. The microstructure investigated by image analysis on specimens extracted from an actual automotive component showed that the injection process induces a strong specific orientation of the fibers according to the Mold Flow Direction (MFD). Moreover, the fiber orientation distribution has been determined quantitatively. Most of the fibers are in the plane of the structure and oriented according to the MFD. For the overall behavior, it has been demonstrated that the tensile behavior of the short fiber reinforced EPC matrix composite is strain rate dependent. In fact, mechanical characteristics, in terms of damage threshold and ultimate properties, are highly sensitive to the strain rate. As the strain rate increases, noticeable effects consist of an increase of the Young's modulus of $50 \%$ and a delayed damage onset. These are followed by a slightly reduced damage accumulation. A significant increase of the damage stress (500\%) and strain (200\%) threshold have been also noticed.

The origin of these variations has been investigated by means of a multi-scale analysis. The objective was to relate the observed macroscopic strain rate effect to the microscopic deformation and damage mechanisms. Interrupted tensile tests have been performed at different rates in order to establish qualitative and quantitative relations between the microscopic phenomena and the macroscopic properties. It has been established that the strain rate effect on the overall behavior and damage is essentially due to the fiber-matrix damage mechanism which propagates through the whole volume of the specimen as well as to matrix micro-cracking. It was established that the strain rate brings about a viscous nature of damage evolution due to the matrix rheology. In fact, it has been shown that the viscoelastic-viscoplastic behavior of the EPC matrix has an important role in the strain rate effect. Microscopic observations have been performed and coupled to interrupted high strain rate tensile tests. For high strain rate, it has been observed that a local zone around the fibers is highly strained due to local matrix rheology. The capability of the matrix to undergo this large strain localization around the fibers explains also the reduced damage $\mathrm{ki}^{-}$ netic observed at the microscopic scale. This statement confirms that this strained zone around the debonded interface dissipates the strain energy and accordingly hinders the interfacial crack propagation through the matrix. It thus reduces the degradation and its kinetics at the microscopic scale. Moreover, the absence of the matrix micro-cracking mechanism can be explained readily by the large matrix macroscopic strain reached for high strain rate. One can conclude that the strain rate effect can be explained by the interaction of two main phenomena: the local and the global viscous behavior of the matrix and fiber-matrix interface debonding. These two phenomena are coupled and lead to: an increase of the composite stiffness, a delay of the damage threshold, a diminution of the fiber-matrix interface failure kinetic and the delay and reduction of the matrix micro-cracking. The comparison of damage kinetics at microscopic and macroscopic levels for quasi-static and dynamic loading confirms that the micro damage indicator is more relevant than the macroscopic stiffness reduction for investigating damage accumulation. Indeed, the macroscopic damage investigation based on the longitudinal Young's modulus reduction cannot reflect the effect of the strain rate on the damage kinetic. Finally, the proposed multi-scale analysis highlights clearly that the strain rate effect observed at the macroscopic scale is especially controlled by the interfacial debonding of disoriented fibers and the EPC matrix viscous rheology. The latter affects the deformation and the damage history at the microscopic scale. In addition, the time-dependent damaged-behavior can explain readily the accommodation exhibited at the macroscopic scale.

In a further paper all the experimental findings, established in this work, will be implemented into a predictive micromechanical model [32,33]. This multi-scale model would integrate fiber-matrix interface damage and the viscous rheology of the thermoplastic matrix in relation with the composite material microstructure.

\section{Acknowledgement}

This work was sponsored by FAURECIA Automotive Exterior, Audincourt, France.

\section{References}

[1] Hufenbach W, Adam F, Beyer J, Zichner M, Krahl M, Lin S, et al. Development of an adapted process technology for complex thermoplastic lightweight structures based on hybrid yarns. In: Proceedings of ICCM-17 conference. Edinburgh; July 2009. p. 27-31.

[2] Schade M, Diestel O, Cherif Ch, Krahl M, Hufenbach W, Franeck J, et al. Development and technological realization of complex shaped textilereinforced thermoplastic composites. In: Proceedings of composites in automotive and aerospace (5th international congress on composites), Munich; October 2009. p. 14-5.

[3] Long AC, Wilks CE, Rudd CD Experimental characterisation of the consolidation of a commingled glass/polypropylene composite. Compos Sci Technol 2001;61:1591-603.

[4] Tufail M. Processing investigation and optimization for hybrid thermoplastic composites. J Univer Sci Technol Beijing 2007;14:185-9.

[5] Tornqvist R, Baser B. Structural modules with improved crash performance using thermoplastic composites. SAE Trans 2002:603-13.

[6] Automotive supplement. Reinf Plast 2003;47(2).

[7] Griffiths B. Glass-reinforced thermoplastic succeeds in car crash structure. Compos Technol 2006:52-4.

[8] Brooks R. Composites in automotive application design. Comprehen Compos Mater 2000;6:341-63.

[9] Ramakrishna S. Microstructural design of composite materials for crashworthy applications. Mater Des 1997;18:167-73.

[10] Mamalis AG, Robinson M, Manolakos DE, Demosthenous GA, Ionnidis MB, Carruthers J. Review - Crashworthy capability of composite material structures. Compos Struct 1997;37:109-34.

[11] Fitoussi J, Meraghni F, Jendli Z, Hug G, Baptiste D. Experimental methodology for high strain-rates tensile behaviour analysis of polymer matrix composites. Compos Sci Technol 2005;65(14):2174-88.

[12] Jendli Z, Meraghni F, Fitoussi J, Baptiste D. Micromechanical analysis of strain rate effect on damage evolution in sheet molding compound composites. Compos Part A: Appl Sci Manuf 2004;35(7-8):779-85.

[13] Shokrieh MM, Omidi MJ. Investigation of strain rate effects on in-plane shear properties of glass/epoxy composites. Compos Struct 2009;91(1):95-102.

[14] Okoli OI, Abdul-Latif A. Failure in composite laminates: overview of an attempt at prediction. Compos Part A: Appl Sci Manuf 2002;33(3):315-21.

[15] Evci C, Gülgeç M. An experimental investigation on the impact response of composite materials. Int J Impact Eng 2012;43:40-51.

[16] Dasari A, Rohrmann J, Misra RDK. Microstructural evolution during tensile deformation of polypropylenes. Mater Sci and Eng: A 2003;351(1-2):200-13. 
[17] Jerabek M, Major Z, Renner K, Móczó J, Pukánszky B, Lang RW. Filler/matrixdebonding and micro-mechanisms of deformation in particulate filled polypropylene composites under tension. Polymer 2010;51(9):2040-8.

[18] Sarang S, Misra RDK. Strain rate sensitive behavior of wollastonite-reinforced ethylene-propylene copolymer composites. Mater Sci Eng: A 2004;381(12):259-72.

[19] Chen Y, Ghosh S. Micromechanical analysis of strain rate-dependent deformation and failure in composite microstructures under dynamic loading conditions. Int J Plast 2012;32-33:218-47.

[20] ABAQUS, ABAQUS/Explicit User's Manual, Version 6.5, Providence, Rhode Island, USA.

[21] Pardo S, Baptiste D, Décobert F, Fitoussi J, Joannic R. Tensile dynamic behaviour of a quasi-unidirectonal E-glass/polyester composite. Compos Sci Technol 2002;62(4):579-84.

[22] Jendli Z, Fitoussi J, Meraghni F, Baptiste D. Anisotropic strain rate effects on fiber-matrix interface decohesion in sheet moulding compound composites. Compos Sci Technol 2005;65(3-4):387-93.

[23] Reis JML et al. Tensile behavior of glass/epoxy laminates at varying strain rates and temperatures. Compos Part B: Eng 2012;43(4):2041-6.

[24] Schoßig M, Bierögel C, Grellmann W, Mecklenburg T. Mechanical behavior of glass-fiber reinforced thermoplastic materials under high strain rates. Polym Testing 2008;27(7):893-900.

[25] Hashemi S. Temperature, strain rate and weldine effects on strength and micromechanical parameters of short glass fibre reinforced polybutylene terephthalate (PBT). Polym Testing 2011;30(8):801-10.
[26] Pardo S. Phd thesis of ENSAM. Thoughness of composites assemblies under dynamic solicitation; 2001 [in French].

[27] Xiao X. Dynamic tensile testing of plastic materials. Polym Testing 2008;27(2): $164-78$.

[28] Fitoussi J, Guo G, Baptiste D. A statistical micromechanical model of anisotropic damage for S.M.C. composites. Compos Sci Technol 1998;58(5): 75963.

[29] Karjcinovic D. Selection of damage parameter - art or science? Mech Mater 1998;28:165-79.

[30] Meraghni F, Desrumaux F, Benzeggagh ML. Implementation of a constitutive micromechanical model for damage analysis in glass mat reinforced composites structures. Compos Sci Technol 2002;62:2087-97.

[31] Margueres Ph, Meraghni F, Benzeggagh ML. Comparison of stiffness measurements and damage investigation techniques for a fatigued and postimpact fatigued GFRP composite obtained by RTM process. Compos A Appl Sci Manuf 2000;31(2):151-63.

[32] Jendli Z, Meraghni F, Fitoussi J, Baptiste D. Multi-scale modelling of dynamic behaviour for discontinuous fiber SMC composites. Compos Sci Technol 2009;69:97-103.

[33] Yang BJ, Kim BR, Lee HK. Predictions of viscoelastic strain rate dependent behavior of fiber-reinforced polymeric composites. Compos Struct 2012;94(4):1420-9. 